\title{
Three methods for measuring range of motion while wearing protective clothing: A comparative study
}

\author{
Paul S. Adams ${ }^{a}$ and W. Monroe Keyserling ${ }^{b}$ \\ " Department of Health Sciences, Illinois State University, Normal, IL 61790-5220, USA. \\ ${ }^{b}$ Center for Ergonomics, Department of Industrial and Operations Engineering, University of Michigan, Ann Arbor, MI 48109-2117, \\ USA
}

Received April 9, 1993; accepted in revised form June 1, 1993

\begin{abstract}
This paper evaluates three methods for objectively measuring ROM while workers are wearing protective clothing: a universal goniometer, a Leighton Flexometer, and an electrogoniometer. Ten male subjects performed a set of nine gross body movements while semi-nude and while wearing each of nine configurations of coveralls. Coveralls varied in relative size and fabric weight. Changes in joint angles were measured simultaneously with the goniometer and the electrogoniometer, and then simultaneously with the Flexometer and the electrogoniometer.

The goniometer and Flexometer data were positively correlated across garment treatments, but the electrogoniometer data were not highly correlated with either the goniometer or Flexometer data. Precision was similar between the goniometer and Flexometer, but the goniometer was found to be much less invasive than the Flexometer.
\end{abstract}

\section{Relevance to industry}

Range-of-motion is an important performance measure for workers wearing personal protective clothing. It is frequently used to compare competing garment designs and to assess worker mobility. This paper compares three methods for objectively measuring range-of-motion and recommends a method for use in future studies.

\section{Keywords}

Range-of-motion; Mobility; Protective clothing; Performance measurement; Goniometer; Leighton Flexometer; Electronic goniometer

\section{Introduction}

Personal protective clothing (PPC) enables many people to work safely in hazardous environments. Unfortunately, PPC may inhibit worker efficiency, resulting in longer task completion times and increased strain on the employee. If the effects of PPC on task performance can be quantified, then models could be developed that

Correspondence to: Paul S. Adams, Ph.D., 103 Moulton Hall, 5220 Illinois State University, Normal, IL 61790-5220, USA. would assist PPC manufacturers in making garment improvements and aid users in garment selection and task scheduling.

PPC effects on performance are typically quantified by measuring physiological or thermal strain, energy expenditure, mobility, task performance efficiency, and psychophysical response. Physiological strain or heat stress studies rely on measurements of heart rate, rectal and skin temperatures, blood pressure, respiration rate and sweat loss (e.g., Bishop et al., 1989; Faff et al., 1989; Vegthe, 1989). Energy expenditure is often determined from oxygen uptake data taken dur- 
ing exercise (e.g., Maxfield et al., 1967). Mobility studies assess the wearer's ability to perform routine movements and typically measure range of motion (ROM) or reach capability (e.g., Huck, 1991; Alexander and Laubach, 1973). When the primary interest is to test wearer performance on a specific task, test batteries, obstacle courses, and simulated tasks are often developed that enable comparison of task completion times (e.g., Lotens, 1986; Dunlap and Associates et al., 1965; Wick et al., 1984). Finally, psychophysical responses measure comfort, wearability, fatigue, perceived exertion, and preferences among competing garments (e.g., Hollies et al., 1979; Gamberale, 1985).

A variety of experimental paradigms have been used to objectively assess mobility restriction caused by protective clothing. Saul and Jaffe (1955) developed a test battery of simple movements and measurements that could be performed with minimal training and equipment. Nicoloff (1957) used an arm and shoulder harness to simulate clothing restriction on upper body movement. Alexander and Laubach (1973) measured arm reach capability in their study on the effect of cold weather flight gear. More recent studies have measured encumbrance using goniometers (Bensel et al., 1987; Bachrach and Egstrom, 1974), Leighton Flexometers (Huck, $1988 ; 1991)$, and a potentiometer-driven microcomputer system (Gregoire et al., 1985).

Among the measures of mobility restriction, range of motion (ROM) has two obvious advantages. First, ROM can be objectively quantified in terms of joint angles or reach distances. Joint angles are typically measured as movement occurring in a single plane and with the joint isolated, i.e., all movement is effected by the single joint. Reach distances often involve more complex motions, with movement occurring in multiple planes and involving multiple joints, such as the twisting of the torso in addition to shoulder flexion. Whereas joint angle measurement is well-suited for use in the laboratory, reach distances directly quantify performance capability for one aspect of an actual task.

A second advantage of using ROM measurements is that they can be taken with instruments that are relatively unsophisticated and easy to use. Typical equipment includes a tape measure and a goniometer or Flexometer, none of which require calibration, installation, or set-up. Training requirements are minimal and the instruments can easily be carried to any location. Methods used to measure energy expenditure (e.g. calorimetry) or physiological strain (e.g. skin thermisters and rectal probes) do not share these advantages.

ROM measurement also has some drawbacks. Tasks often involve movements that cannot be measured by a single instrument or technique. In addition, PPC complicates angle measurement by hiding body landmarks and joint centers. Even when joints are fully exposed, joint angle readings may not agree among different observers and types of instruments (Goodwin et al., 1992).

While several studies have compared clinical methods of goniometry (e.g., Petherick et al., 1988; Goodwin et al., 1992), they have typically measured performance at only one or two joints. Relatively few studies have compared methods for assessing the effects of PPC on range-of-motion (Adams, 1993; Gregoire et al., 1985; Huck, 1991).

The principal motivations for the study described below were as follows:

- To select a ROM measurement method for use in studies of PPC effects on worker performance

- To determine if previously reported methods introduce measurement artifacts; i.e., whether these methods may themselves interfere with ROM when measuring clothed subjects

- To evaluate an electrogoniometric system that is capable of being worn underneath clothing. The primary objective of this study was to compare three methods, a universal goniometer, a Leighton Flexometer, and an electrogoniometer, for measuring range-of-motion of subjects wearing protective clothing. A secondary objective was to determine if measurements were affected by garment characteristics, since Adams (1993) demonstrated that garment size can affect ROM.

\section{Criteria for comparison}

The following criteria were proposed by Chaffin and Andersson (1991) as appropriate for com- 
paring ROM measurement methods: accuracy, repeatability (precision), ease of use, cost, and measurement flexibility. In addition, measurement systems should be safe, non-invasive, and able to withstand the rigors of laboratory and field testing. Most of these criteria are self-explanatory and will be included in a comparison summary presented later in this paper.

Four of the above criteria are especially relevant for ROM studies involving PPC: measurement flexibility, invasiveness, accuracy and re- peatability. First, a good test method is flexible and able to measure a variety of movements under a wide range of test conditions. Second, a good test method allows a subject to move without interference from instrumentation and peripheral equipment. Tests should measure only the parameter of interest and be free from confounding artifacts. For example, arm reach distances will be overstated if shoulder locations are not stabilized, and length-of-stride measurements may be affected by striations in walking surfaces.

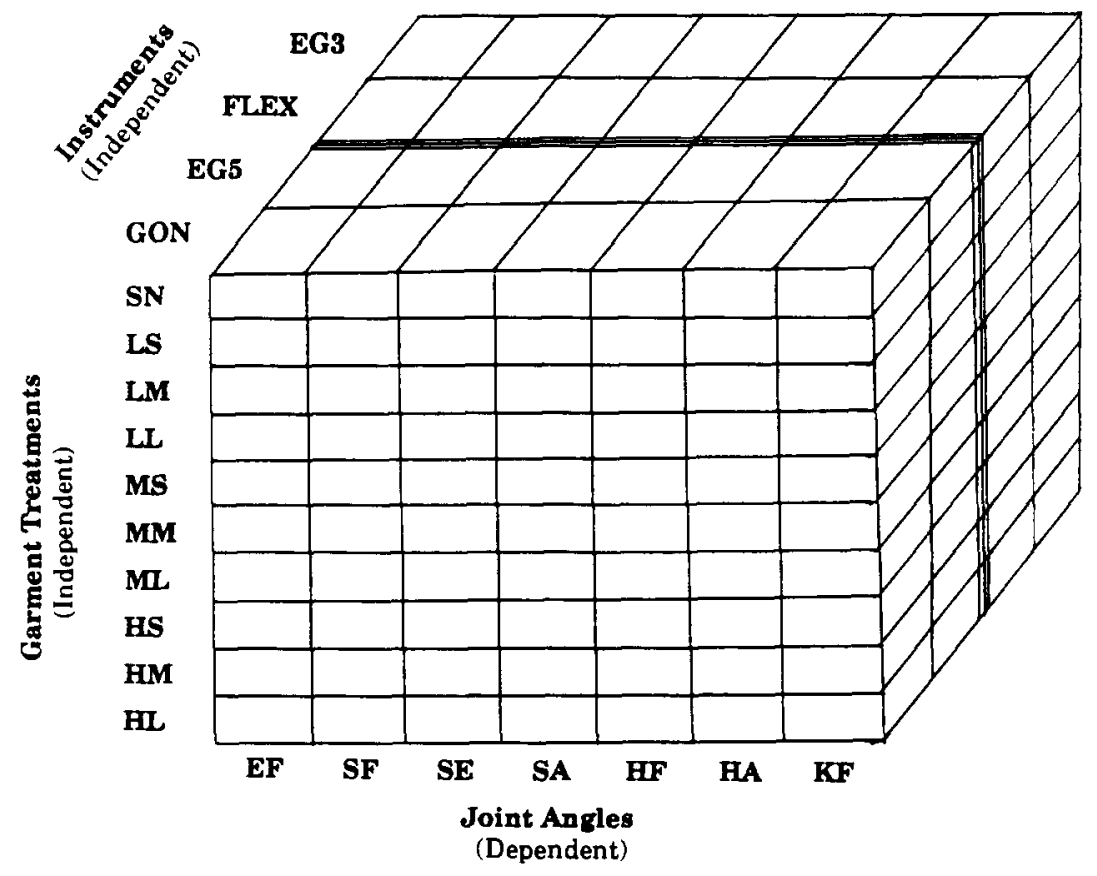

KaY:

\section{Garment Conditions}

SN - semi-nude

LS - light-weight fabric, undersized

LM - light-weight, appropriately sized

LL - light-weight, oversized

MS - medium-weight, undersized

MM - medium-weight, appropriately sized

ML - medium-weight, oversized

HS - heavy-weight, undersized

HM - heavy-weight, appropriately sized

HL - heavy-weight, oversized

\section{Datasets}

GON - goniometer data, 5 trials per condition

EG5 - electrogoniometer data, 5 trials per condition

FLEX - flexometer data, 3 trials per condition

EG3 - electrogoniometer data, 3 trials per condition

\section{Movement.}

EF - elbow flexion

SF - shoulder flexion

SE - shoulder extension

$\mathrm{SA}$ - shoulder abduction

HF - hip flexion

HA - hip abduction

KF - knee flexion

Note: Data sets GON and EG5 were collected simultaneously, as were FLEX and EG3.

Fig. 1. Experimental design for comparative study of three range-of-motion measurement methods. 


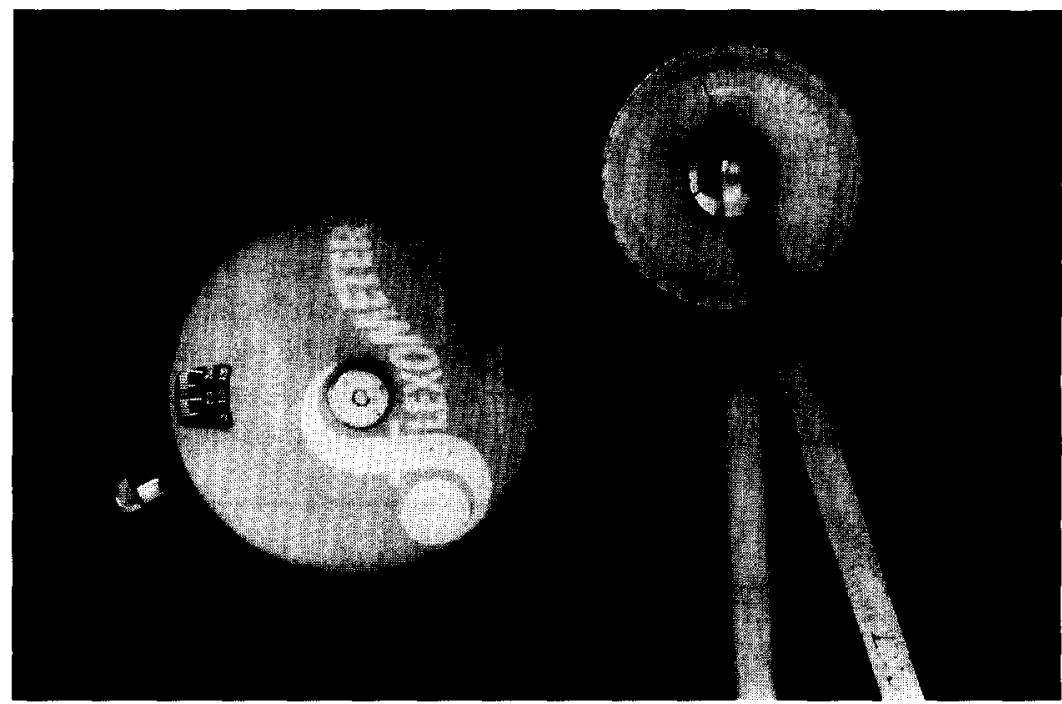

Fig. 2. Photograph of a Leighton Flexometer and a universal goniometer.

Finally, good test methods yield data that are true (accurate) recordings of the measure of interest and have minimal variance among identical events.

\section{Method}

\subsection{Experimental design}

The independent variables were measurement instrument, garment condition and type of movement. The dependent variables were joint angles as measured by each of three test instruments. The experimental design was a full factorial $4 \times$ $10 \times 7$ design with replicates, as shown in Fig. 1 . Each subject performed a full set of seven movements in each of ten garment treatment conditions, including a semi-nude condition. Two additional movements, shoulder horizontal flexion and extension, were also performed using only two of the instruments. (These two movements were included because of their importance in PPC design; however, range-of-motion measurement in the horizontal plane could not be done with the Leighton Flexometer.) The order of the treatment conditions was systematically sequenced across subjects. Since it was impossible to collect data simultaneously from all three of the instruments being evaluated, joint angles were mea- sured in paired sets as described below, yielding four sets of data.

\subsection{Apparatus}

The three instruments selected for testing were a universal or manual full-circle goniometer, a Leighton Flexometer, and an electrogoniometer. The universal goniometer (J. Skylar Mfg. Co.), shown in Fig. 2, is widely used by clinicians for ROM measurement. It consists of two movable stainless steel arms that pivot about the center of a full-circle steel protractor. To measure joint angles, the pivot pin is positioned at the joint center and the goniometer arms are aligned with the adjoining body segments.

The Leighton Flexometer, also shown in Fig. 2 , is similar to that used by Huck $(1988,1991)$ in her studies of fire fighter PPC restriction. It consists of two weighted dials that move independently within a case, and a pair of manually operated brakes. The device is strapped to a subject's limb and one dial is locked as the subject assumes the beginning posture. The subject then moves and the second dial is locked at the extent of motion. Range of motion is taken as the difference between the two readings.

The electrogoniometer system is shown in Fig. 3. The sensors for this system (Penny and Giles Biometrics Ltd., Gwent, UK) utilize a set of strain 


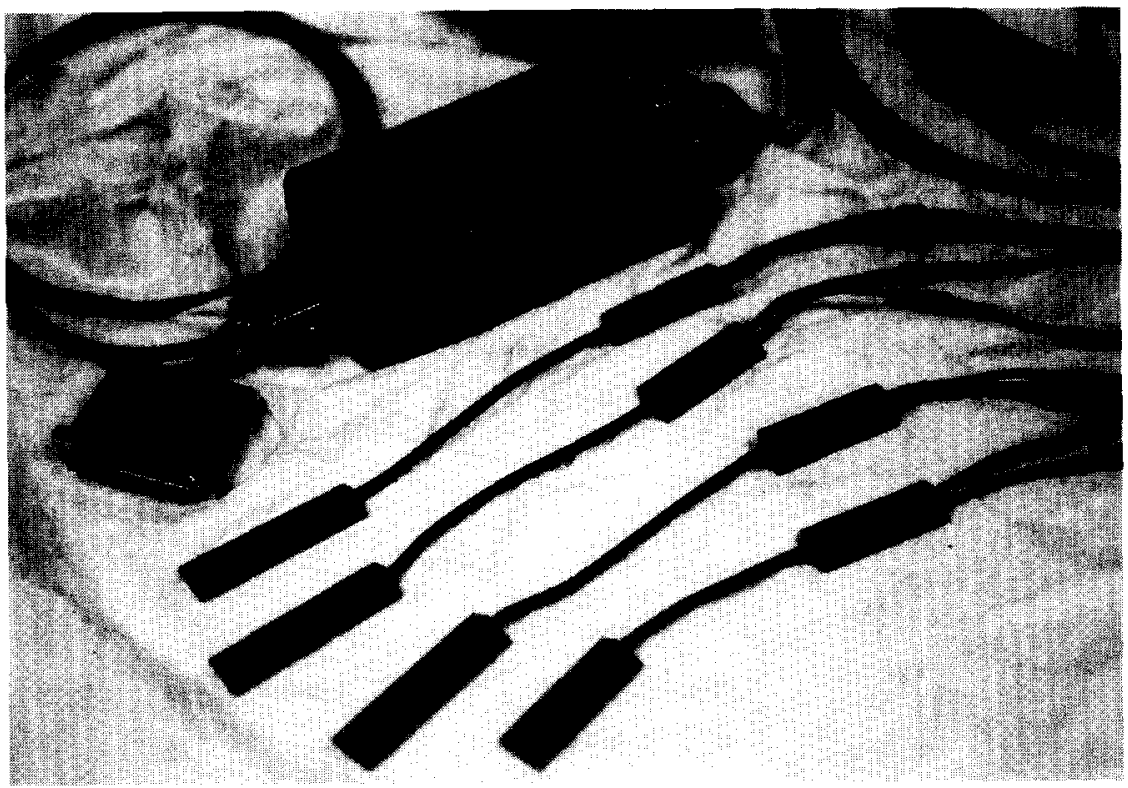

Fig. 3. Photograph of electrogoniometer system, including sensors and leads (Penny and Giles, Ltd., Gwent, UK) and 8-channel amplifier (University of Michigan Center for Ergonomics, Ann Arbor, MI.)

gauges mounted within a protective spring. End blocks are attached to the subject's skin on either side of a joint by double-backed tape. The strain gauges are wired to an amplifier and microcomputer. The amplifier and software were developed at the University of Michigan's Center for Ergonomics (1992). The system measures joint angles in two orthogonal planes to within $\pm 1 \frac{1}{2}^{\circ}$.

Most movements in this study were performed so that a single channel or plane was involved. For movements across two planes, a geometrical algorithm was used to integrate angle readings into a single value for change in joint angle.

\subsection{Subjects}

Ten healthy males participated, ranging in age from 19 to 31 years. Heights ranged from 173.1 to $187.5 \mathrm{~cm}$ (37th to 96th percentile for U.S. civilians), and weights from 145.6 to $207.5 \mathrm{lbs}$. (19th to 87 th percentile) (Abraham, 1979).

\subsection{Test garments}

The ten garment treatments included a seminude condition and nine test garments. Semi-nude trial apparel consisted of athletic shorts, briefs, socks, and athletic shoes. Treatment garments were long-sleeved coveralls, which were worn over the semi-nude trial clothing. The coveralls had a zippered front with a covering flap, a snap at the top of the zipper and at the waist, set-in sleeves with sriaps at the wrists, an elasticized waist, and two expansion pleats running vertically down either side of the back from the top of the shoulder to the waist.

The coveralls were sewn by a single manufacturer (Lion Apparel, Dayton, $\mathrm{OH}$ ) from each of three woven fabrics using consistent patterns. The fabrics were $4.25 \mathrm{oz} / \mathrm{yd} 265 / 35$ Poly/Cotton, $7.25 \mathrm{oz} / \mathrm{yd}^{2} 50 / 50$ Poly/Cotton, and $10.0 \mathrm{oz} / \mathrm{yd}^{2}$ $50 / 50$ Poly/Cotton. Each subject wore three sizes of coveralls:

- an appropriately sized set (Mediums or Larges),

- an undersized set (Smalls or Mediums),

- an oversized set (Extra Larges).

Appropriate size was determined from the manufacturer's recommended sizing chart, based on height and weight. Fabric weight and size were not treated as separate factors since Adams (1993) found fabric weight to have minimal effect on ROM.

All trials were conducted in a laboratory under ambient conditions of approximately $20^{\circ} \mathrm{C}$ and $50 \%$ relative humidity. 


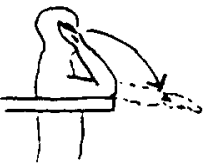

Elbow Flexion

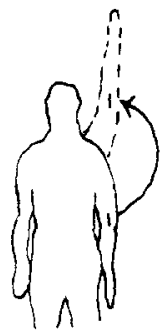

Shoulder Abduction

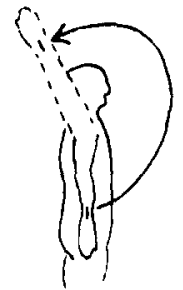

Shoulder Flexion

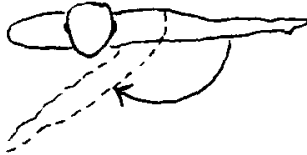

Shoulder Horizontal Flexion

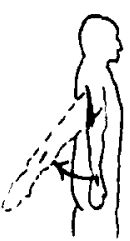

Shoulder Extension

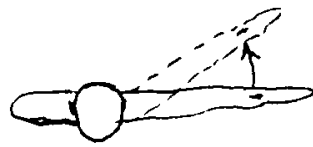

Shoulder Horizontal Extension

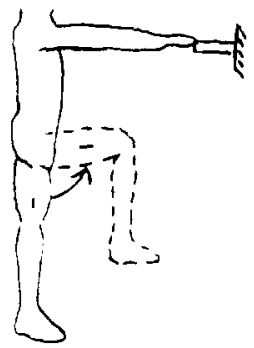

Hip Flexion

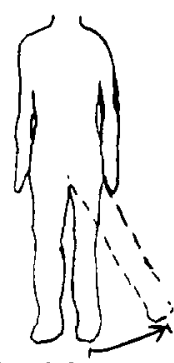

Hip Abduction

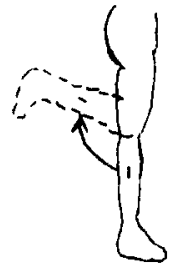

Knee Flexion

Fig. 4. Range-of-motion movements performed and angles measured. (Adapted from Huck, 1988)

\subsection{Movements}

Test movements were adapted from Saul and Jaffe (1955) and Huck (1988) and are illustrated in Fig. 4. Those movements measured with all three instruments included elbow flexion, shoulder flexion, shoulder extension, shoulder abduction, hip flexion, hip abduction, and knee flexion. Shoulder horizontal flexion and extension were only measured with the goniometer and electronic goniometer, since these motions are performed horizontally and could not be measured by the Flexometer

\subsection{Test procedure}

Anthropometric data were collected from each subject prior to testing and were used in selecting a set of nine test garments. The subject performed a pre-established set of stretching exercises to loosen shoulder, torso, hip and leg muscles.

Electrogoniometer sensors were then mounted across the right elbow, hip, and knee joints according to the manufacturer's instructions. A fourth sensor was placed across the shoulder, with one end mounted on the dorsal scapular 
surface and the other on the dorsal surface of the right upper arm. The two sensor ends were thus aligned when the arm was abducted in the coronal plane parallel to the floor. Electrical leads were secured with tape.

The test procedure began with the subject donning the specified test garment and closing all fasteners. The subject was asked to perform each of nine movements five times, each time moving "as far as you can without straining". Joint angles were measured simultaneously with the goniometer (data set GON) and the electrogoniometer system (data set EG5).

After completing the first set of nine movements, the subject was asked to perform a second set of seven movements. (Shoulder horizontal flexion and extension could not be measured with a Flexometer.) Three repetitions were made of each movement while Flexometer (data set FLEX) and electrogoniometer data (data set EG3) were simultaneously collected.

All ROM goniometer and Flexometer measurements were taken by the same tester. This precluded the well-documented problem of inter-tester reliability (Petherick et al., 1988; Mayerson and Milano, 1984; Boone et al., 1978).

All measurements for a single garment condition were collected prior to taking the garment off. The movement sequence (beginning with elbow flexion and ending with knee flexion) was

\section{Grand Means of Angle Measurements}

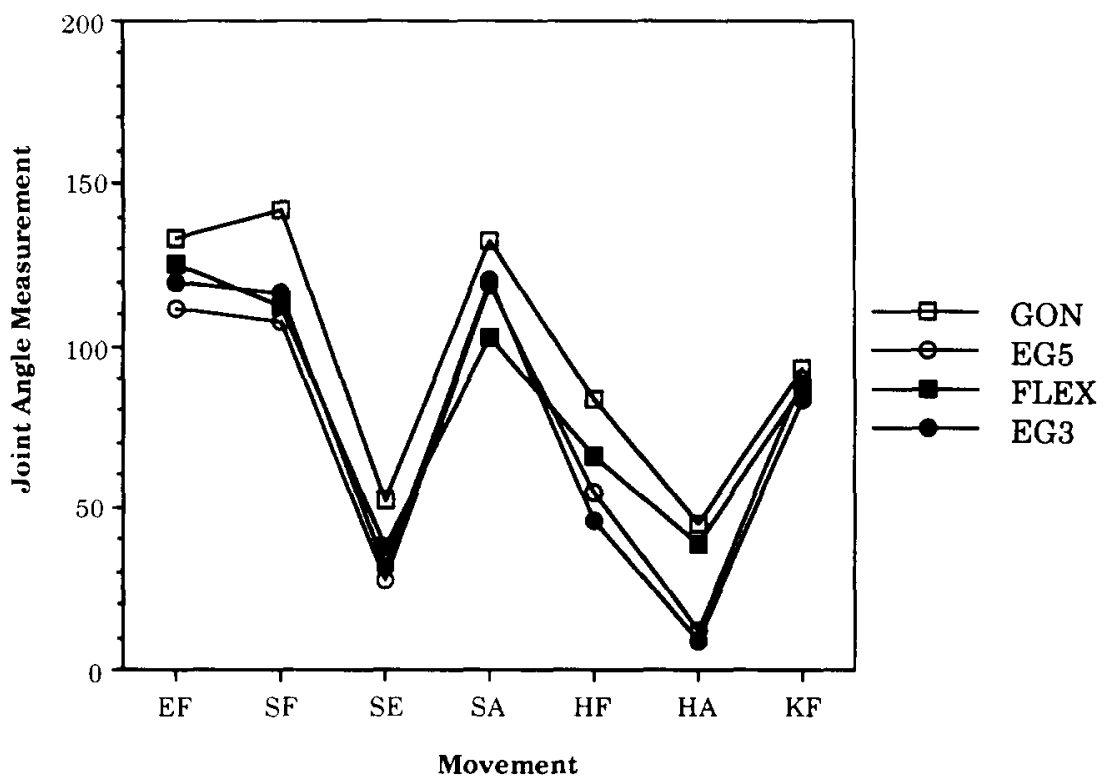

KEX:

\section{Mevements}

EF - elbow flexion

$\mathrm{SE}$ - shoulder extension

HF - hip flexion

KF - knee flexion

SF - shoulder flexion

SA - shoulder abduction

HA - hip abduction

\section{DataSets}

GON - goniometer data, 5 trials per condition

EG5 - electrogoniometer data, 5 trials per condition

FLEX - flexometer data, 3 trials per condition

EG3 - electrogoniometer data, 3 trials per condition

Fig. 5. Grand means of four data sets by movement. 
maintained for all conditions and subjects. Movements were performed slowly to facilitate measurement and at no time was perspiration visible on any of the subjects. Each subject completed all trials in a single testing session, with a lunch/ dinner break of approximately one hour taken near the midpoint.

\subsection{Data analysis}

Pearson correlations were calculated between simultaneously collected data sets (e.g. GON and EG5), both for all garment treatments using SAS software (SAS Institute Inc., 1988), and within each garment condition using the SYSTAT statistical package (Systat, Inc., 1987). Means and standard deviations for each subset of five (or three) replicates within a cell were calculated using spreadsheet software (Lotus Development Corporation, 1986). To test for homogeneity of the data across garment treatments, instruments and movements, analysis of variance (ANOVA) was performed on these standard deviations using the BMDP.2V statistical package.

\section{Results}

\subsection{Correlation among instruments}

Fig. 5 shows the grand means of the four data sets by movement. In general, large GON angles corresponded to large EG5 angles (e.g., elbow flexion and shoulder abduction), and likewise for angles in the FLEX and EG3 data sets. Although this result suggested strong agreement among measurements from all three instruments, correlations within movements found otherwise.

Coefficients of determination $\left(r^{2}\right)$ were computed between the simultaneous angle measurements in the goniometer (GON) and electrogoniometer (EG5) data sets, and between the Flexometer (FLEX) and electrogoniometer (EG3) data sets for all movements and conditions. Within movements, the comparatively small changes in angles among trials were not well correlated between GON and EG5, or between FLEX and EG3.

Since goniometer and Flexometer data could not be collected simultaneously, and because
Table 1

Coefficients of Determination $\left(r^{2}\right)$ between Goniometer (GON) and Flexometer (FLEX) data sets as calculated from means of trial subsets. Comparable coefficients between the simultaneously collected data sets (GON and EG5; FLEX and EG3) are also shown. $r^{2}$ values associated with negative correlations are indicated by asterisks $\left({ }^{*}\right)$

\begin{tabular}{|c|c|c|c|}
\hline Movement & $\begin{array}{l}\text { GON/ } \\
\text { FLEX }\end{array}$ & $\begin{array}{l}\text { GON/ } \\
\text { EG5 }\end{array}$ & $\begin{array}{l}\text { FLEX/ } \\
\text { EG3 }\end{array}$ \\
\hline Elbow flexion & 0.339 & 0.268 & 0.397 \\
\hline Shoulder flexion & 0.785 & $0.306 *$ & 0.055 \\
\hline Shoulder extension & 0.268 & ns & $0.044 *$ \\
\hline Shoulder abduction & 0.760 & $0.437^{*}$ & 0.059 \\
\hline Shoulder horizontal flexion & NA & 0.101 & NA \\
\hline Shoulder hori. extension & NA & ns & NA \\
\hline Hip flexion & 0.738 & ns & $\mathrm{ns}$ \\
\hline Hip abduction & 0.799 & $0.397 *$ & $0.299 *$ \\
\hline Knee flexion & 0.397 & 0.067 & 0.089 \\
\hline Overall ( $r^{2}$ for means) ${ }^{a}$ & 0.897 & 0.279 & 0.127 \\
\hline
\end{tabular}

ns - Not significant $(p \leq 0.05)$

NA - Not applicable. Shoulder horizontal flexion and extension cannot be measured with a Leighton Flexometer.

it Overall $r^{2}$ values reflect the fact that all three measurement systems yield large angle measurements for large joint angles (e.g., elbow flexion), and small readings for smaller joint angles (e.g., hip abduction). Therefore, the coefficients of determination across all movements are highly positive.

these sets contained five and three trials per subject-condition respectively, trial means were compared for each subject-condition. The overall coefficient of determination $\left(r^{2}\right)$ between the GON and FLEX data set means was 0.897. Additional $r^{2}$ values between GON and FLEX are shown in Table 1 for each of the seven shared movements, along with comparable $r^{2}$ values for trial means between GON and EG5, and between FLEX and EG3.

\subsection{Measurement differences among methods}

An important criterion for ROM measurement methods is how well the instruments agree on measurements of change in joint angles. Table 2 shows the grand mean differences by movement between data sets. Goniometer measurements of ROM were consistently larger than Flexometer measurements, although the discrepancy varied with movement. Goniometer and Flexometer measurements were both larger than values recorded by the electrogoniometer. 
Table 2

Grand mean differences between GON, FLEX, EG5, and EG3 over all trials, garment treatments and subjects (in degrees)

\begin{tabular}{lclc}
\hline Movement & \multicolumn{3}{c}{ Differences between grand means } \\
\cline { 2 - 4 } & GON- & $\begin{array}{l}\text { GON- } \\
\text { EG5 }\end{array}$ & $\begin{array}{l}\text { FLEX- } \\
\text { EG3 }\end{array}$ \\
\hline Elbow flexion & 8 & 22 & 5 \\
Shoulder flexion & 30 & 35 & -3 \\
Shoulder extension & 15 & 25 & 6 \\
Shoulder abduction & 30 & 14 & -18 \\
Hip flexion & 18 & 29 & 20 \\
Hip abduction & 6 & 33 & 30 \\
Knee flexion & 6 & 3 & 4 \\
\hline
\end{tabular}

Fig. 6 shows a plot of the overall means for a typical movement, hip flexion. Trends in the data are generally consistent across the ten garment treatments between the goniometer and Flex-
Table 3

Means of Standard Deviations $(S D)$ for all movements and measurement methods (in degrees)

\begin{tabular}{lllll}
\hline Movement & \multicolumn{3}{l}{ Data set } \\
\cline { 2 - 5 } & GON & EG5 & FLEX & EG3 \\
\hline Elbow flexion & 2.0 & 4.4 & 2.8 & 2.4 \\
Shoulder flexion & 5.3 & 3.3 & 3.1 & 7.8 \\
Shoulder extension & 3.0 & 2.9 & 2.7 & 2.2 \\
Shoulder abduction & 5.0 & 2.8 & 2.9 & 5.1 \\
Shoulder horizontal flexion & 3.7 & 3.1 & na & na \\
Shoulder hori. extension & 3.2 & 3.3 & na & na \\
Hip flexion & 3.8 & 3.4 & 2.4 & 3.0 \\
Hip abduction & 3.1 & 2.7 & 2.1 & 2.0 \\
Knee flexion & 4.3 & 3.4 & 2.9 & 3.4 \\
\hline
\end{tabular}

na - not applicable

Note: The standard deviation for the electronic goniometer data sets (EG5 and EG3) were computed after numerous data points had been descarded. (Criteria for discarding data are discussed in section 5.)

\section{Hip Flexion Means by Garment Treatment}

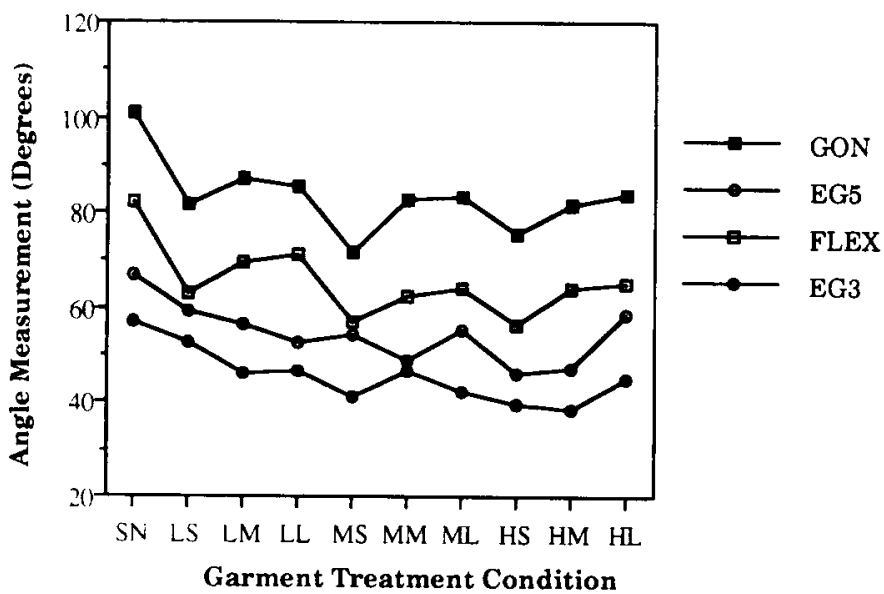

KEY:

Garment Treatment Condition

\section{Garment Conditions}

SN - semi-nude

LS - light-weight fabric, undersized

LM - light-weight fabric, appropriately sized

LL - light-weight fabric, oversized

MS - medium-weight fabric, undersized
MM - medium-weight fabric, appropriately sized

ML - medium-weight fabric, oversized

HS - heavy-weight fabric, undersized

HM - heavy-weight fabric, appropriately sized

HL - heavy-weight fabric, oversized

\section{DataSets}

GON - goniometer data, 5 trials per condition EG5 - electrogoniometer data, 5 trials per condition FLEX - flexometer data, 3 trials per condition EG3 - electrogoniometer data, 3 trials per condition

Fig. 6. Hip flexion means by garment condition. 
Table 4

Analyses of variance (ANOVA's) performed on standard deviations, i.e., standard deviation of five (or three) trials within each treatment/movement/subject condition

\begin{tabular}{lcrrr}
\hline Source & Mean squares & $d f$ & $F$ & $p$-value \\
\hline Goniometer (GON - & movements) & & & \\
Movement & 109.27 & 8 & 16.61 & $<0.001$ \\
Garment & 550.50 & 9 & 83.70 & $<0.001$ \\
Movement-Garment & 46.90 & 2 & 7.13 & $<0.001$ \\
Error & 6.58 & 810 & & \\
Electrogoniometer (EG5 -9 movements) & & \\
Movement & 22.95 & 8 & 6.76 & $<0.001$ \\
Garment & 3.94 & 9 & 1.16 & 0.318 \\
Movement-Garment & 4.96 & 72 & 1.46 & 0.010 \\
Error & 3.39 & 757 & & \\
& & & & \\
Flexometer (FLEX - 7 movements) & & & \\
Movement & 11.41 & 6 & 3.49 & 0.002 \\
Garment & 3.74 & 9 & 1.15 & 0.328 \\
Movement-Garment & 3.58 & 54 & 1.10 & 0.302 \\
Error & 3.26 & 601 & & \\
Electrogoniometer (EG3 & -7 movements) & & \\
Movement & 377.51 & 6 & 28.09 & $<0.001$ \\
Garment & 9.39 & 9 & 0.70 & 0.710 \\
Movement-Garment & 11.38 & 54 & 0.85 & 0.773 \\
Error & 13.44 & 512 & & \\
\hline
\end{tabular}

ometer, but not consistent with the electronic goniometer.

\subsection{Test-retest reliability}

Test-retest reliability for an instrument is reflected by homogeneity among repeated measurements. Standard deviations were computed for each set of five (or three) repeated trials. The means of these standard deviations are presented in Table 3. In general, these standard deviations are slightly larger than intra-tester standard deviations reported by Boone et al. (1978), but slightly smaller than the inter-tester errors they reported. Boone et al. used goniometers and measured angles of joints that were fully exposed, i.e., without clothes.

ANOVA was performed on the standard deviations to test for movement and garment effects on test-retest variability. The results of this analysis are presented in Table 4. Differences in the variability of the data by movement were found to be significant $(p<0.01)$, i.e., test-retest variability was larger for some movements than for oth- ers. Test-retest variability was not significantly affected by the garment treatment ( $p>0.05$ ).

\section{Discussion}

\subsection{Goniometer and Flexometer data}

\subsubsection{Interchangeability of instruments}

Goodwin et al. (1992) found significant differences in readings for elbow range-of-motion among the universal goniometer, fluid goniometer, and electrogoniometer. Hence, they concluded that those instruments should not be used interchangeably. The universal goniometer and electrogoniometer used in Goodwin et al.'s research were very similar to those evaluated in the current study. Our data support the findings of Goodwin et al.; instrument interchangeability is not recommended, based on the discrepancies found among joint angle measurements.

It is encouraging that the goniometer and Flexometer data were positively correlated, given the fact that these readings were not taken simultaneously. This suggests that either of the two instruments could be used in future studies (but not interchangeably) and should lead to similar conclusions regarding garment effects on ROM.

\subsubsection{Measurement differences}

Several sources may contribute to the apparent measurement difference between the goniometer and Flexometer data sets. First, the Flexometer is attached to the subjects' limbs by a circumferential strap that must be fastened snugly to properly secure the instrument. This effectively ties the garment to the subject, preventing it from sliding normally across the skin during movement. Garment slip has been identified as one of the key factors that determines a garment's effect on mobility (Kirk and Ibrahim, 1966). The amount of displacement that normally occurs varies with movement being performed. The fact that the average reduction in $\mathrm{ROM}$ from the semi-nude to oversized garment conditions was 13.1 degrees for the Flexometer, compared to 9.5 degrees for the goniometer (taken across the seven common movements), suggests that the Flexometer may have affected ROM. There was also anecdotal evidence; several of the subjects complained about 
the interference, restriction and discomfort caused by the Flexometer. This artifact may preclude the use of Flexometers in future studies of garment effects on ROM.

Operator error is another possible source of discrepancy between the Flexometer and the goniometer readings. To be accurate, the goniometer must be positioned at a joint such that its arms align with two body segments and the pivot pin is over the axis of rotation. Clothing covered the joint centers in this study, potentially compromising the accuracy of joint center location. While obscured joint centers may have resulted in the higher variance of the clothed conditions over the semi-nude condition $\left(\sigma=3.6^{\circ}\right.$ and $\sigma=2.6^{\circ}$, respectively), it was not apparent from the data whether this problem also contributed to the measurement difference between GON and FLEX.

The Flexometer may also be subject to operator error. Correct operation requires the tester to hold the device and manually set brakes at the beginning and extent of each movement. For an accurate reading, the device should not rotate orthogonally during movement or be moved by the tester. Natural rotation of the garment and underlying body tissue typically resulted in some orthogonal rotation of the dials, despite efforts to reduce this problem through careful instrument placement. The amount of this extraneous rotation appeared to vary with movement being performed. The tester in this study often found it difficult to maintain Flexometer position throughout the movement without interfering with the movement itself. Anecdotal comments from a few of the subjects indicated that this artifact may have been present in some Flexometer trials.

The Flexometer relies on a weighted dial that acts as a pendulum; accurate angle measurement can only occur after the oscillating dial has stabilized. This waiting period between motion termination and measurement, although typically less than a few seconds, may result in subjects reducing the extent of motion to relieve muscular stress.

Finally, both the Flexometer and goniometer have moving parts that are subject to mechanical error from friction. The goniometer has a resistance screw attached at the pivot pin that can be used to lock the arm positions. There were occasions when this screw became tightened during movement and the tester adjusted the goniometer arms against the screw resistance, reducing the speed and perhaps accuracy of measurement. The Flexometer relies on freely moving dials and spring loaded brakes. Excessive resistance at the dial axis would result in angle readings that were too low. It is not known whether instrument error contributed to the observed differences between the goniometer and Flexometer readings.

\subsubsection{Garment effects}

For the GON and FLEX data sets, garment effects appear to follow a consistent trend across garment sizes (see Fig. 6). This finding suggests that these methods yield consistent, repeatable results. Previous ROM studies have also found high test-retest reliability for short inter-test intervals (Goodwin et al., 1992; Rothstein et al., 1983). This is encouraging since replicates are not practical in many PPC studies due to time and physiological constraints, and rest periods are frequently required between trials.

\subsection{Electrogoniometer data}

Correlation coefficients between the electrogoniometer data and the simultaneous goniometer and Flexometer data were best for the elbow and knee joints, and for the semi-nude condition. However the system was generally unreliable, as indicated by the wide variations in correlations (including negative correlations) and the need to discard $7.9 \%$ of the data points from EG5 and $10.3 \%$ from EG3 prior to analysis. See Appendix for criteria used to discard data.

\subsubsection{Insignificant and negative correlations}

Four problems were noted in the collection of electrogoniometric data that may have resulted in the large number of insignificant and negative correlations between the GON and EG5 data sets, and between FLEX and EG3.

(1) The strain gauges (contained within a spring type protective sleeve) frequently became kinked or bent into an " $S$ " shape during certain movements. This was especially common during shoulder extension, shoulder horizontal extension, shoulder abduction, and hip abduction. During these movements, the end blocks of the sensors tended to move toward 
each other, placing the strain gauge in compression rather than tension. When clothing was worn, the compressed gauge was trapped and forced to bend out of the plane of joint movement.

(2) One of the reasons that the manufacturer of the electrogoniometer sensors does not recommend their use at the shoulder is the presence of a third degree of motion at this joint, i.e., humeral rotation (Penny and Giles, Ltd., 1991). Attempts to measure shoulder angles were reported by the manufacturer to be marginally successful, as long as humeral rotation was not involved (Penny and Giles, 1991). The electrogonimeter system was used for shoulder movements in this study to determine whether this system could reliably measure any of the basic test movements, i.e., shoulder flexion, extension, abduction, horizontal flexion and horizontal extension. We observed that the sensor blocks had a tendency to rotate with respect to each other during all shoulder movements, although this rotation appeared to be less than $30^{\circ}$ (estimated) for shoulder extension and shoulder horizontal flexion. The resulting twisting of the strain gauges can introduce "cross-talk", thereby precluding accurate angle measurement (Penny and Giles, Ltd, 1991). The findings of this study support the recommendation of the manufacturer that the type of sensors used in this experiment are not wellsuited to shoulder angle measurement.

(3) The placement of the sensor end blocks on the skin is critical for consistent angle measurement. While placement of the elbow and knee sensors was relatively straightforward, hip sensor placement was more problematic due to two conditions. First, the subjects in this study wore briefs under the test garments. The two sensor blocks were placed on the distal side of the upper leg at the hip and on the side of the torso immediately above the top of the underwear. The elastic band of the underwear crossed over the sensor wires. It was observed that underwear waistbands occasionally contacted the upper sensor, potentially affecting its orientation. The elastic waistbands of the coveralls also moved across the top sensor block.
On several subjects, the top sensor block was placed on fatty tissue near the waistline. During hip abduction, this tissue tends to "roll", causing the attached sensor to move in the same plane as the leg movement. Therefore, the accuracy and reliability of hip abduction and flexion data may have been compromised by differences among subjects in the amount of fatty tissue at the waist, and by vertical adjustments for underwear waistbands. These problems would have had the largest potential effect on measurements of hip abduction.

(4) Those sensors that failed in the course of the testing may have yielded inaccurate readings prior to total failure (and subsequent removal from service). Calibration was only performed prior to sensor attachment, so progressive failure of the strain gauges could not have been detected until total failure was observed. The gauges have a limited life, and since the subjects wore all of the sensors throughout their test period, the gauges were flexed extraneously many times. Future studies with the electrogoniometer system should incorporate frequent calibration procedures and reduce unnecessary strain gauge flexions.

\subsection{Evaluation based on comparison criteria}

Table 5 presents a comparative summary of the three ROM measurement methods with respect to the test method criteria discussed in the Introduction.

\subsubsection{Goniometer}

The goniometer is inexpensive, durable, and can be used to measure many joint angles. Although it is simple to use, the usual method of aligning the instrument with body landmarks is compromised by clothing.

\subsubsection{Flexometer}

The Flexometer is also relatively inexpensive and durable. It is somewhat more difficult to use than the goniometer, and the Flexometer is limited in the number of motions it can measure due to its reliance on gravity. When worn over clothing, the device appears to introduce measurement artifacts. The accuracy and precision of the Flexometer may also be compromised by the displacement of underlying garments. 
Table 5

Comparison criteria summary for three ROM measurement techniques

\section{Cost}

Goniometer: $\$ 42$

Flexometer: $\$ 395$

Electrogoniometer: $\$ 478$ per sensor, plus $\$ 65 \times 2$ cables per sensor, plus amplifier and display or software and microcomputer

\section{Ease of use}

Goniometer: Easy to use; locating limbs and joint centers is compromised by clothing.

Flexometer: Conceptually simple to use; operator must hold device in position and manually set two dial brakes prior to reading. (Holding the device against a moving limb requires awkward postures on the part of the operator and may reduce measurement accuracy.)

Electrogoniometer: Sensor placement is critical; sensors are attached to skin with adhesive. Although considerable set-up is required for each subject, joint angle measurement and data collection are easy once the system is working.

\section{Durability}

Goniometer: Very durable.

Flexometer: Durable.

Electrogoniometer: Very delicate and prone to failure when worn under clothing. Cables can easily be dislodged from sensor leads and leads can even be pulled out of sensor blocks, destroying the sensor. Life limitation of approximately 10,000 flexions (Penny and Giles, 1992). (In this study, only one of four original sensors had both channels functioning after testing 10 subjects in the laboratory. This sensor was used for the hip and shoulder joints on the final subject. Two sensors continued to have one channel functioning and were placed at the elbow and knee where only one channel was required for angle measurement. One sensor was destroyed when an inadvertent movement resulted in irreparable damage.)

\section{Invasiveness}

Goniometer: Unobtrusive since instrument does not touch subject.

Flexometer: Highly obtrusive when worn over garments and secured with straps. Device is uncomfortable. (The straps tie the clothing to the subjects skin, preventing the garment from sliding across the skin normally. Having the operator holding the device while it is attached to a subject also gives the subject a sense of restriction.)

Electrogoniometer: Relatively unobtrusive, although cables running from the sensors to the amplifier can interfere with subject movement since they must exit the garment at some opening. (Care must be taken in securing cables to the subject to allow freedom of movement without "pulling"' $\Rightarrow$

Flexibility

Goniometer: Can be used to measure most joint angles.
Table 5 (continued)

Flexometer: Device relies on gravity and can only measure angles with respect to vertical. Also not well suited for measuring trunk angles.

Electrogoniometer: Manufacturer only recommends use at finger, wrist, elbow, hip and knee joints. (Use at the shoulder is complicated by shoulder rotation which introduces "cross-talk" between channels, although some limited success in measuring shoulder angles was achieved by placing one sensor block on scapular region and the other on the dorsal surface of the upper arm. Strain gage length can be too short to allow extreme extension. Gage wire can also become kinked or looped during flexion.)

\section{Accuracy}

Goniometer: Depends upon skill of operator to align instrument arms with body segments and to locate joint centers. (Clothing compromises the ability to perform these tasks.)

Flexometer: Depends upon skill of operator in minimizing interference with subject movement and in reading the dials. Also affected by how well the instrument's position is maintained with respect to the limb during movement. (The weighted dials tend to oscillate with movement and reading should only be taken after dials have stabilized. This may require the subject to hold a posture at the limits of "comfortable movement" for several seconds.)

Electrogoniometer: Sensor placement is critical. Reasonable agreement with other instruments for unclothed elbow and knee angle measurements. Extremely poor results for all other conditions and joints. (Numerous data points were missed due to open connections or other hardware problems.)

\section{Precision}

Goniometer: Depends upon ability of operator to consistently locate landmarks.

Flexometer: Depends upon operator skill in handling device during movement and in dealing with oscillations.

Electrogoniometer: Appears to be similar to, or slightly better than, other two instruments tested. Data must be carefully screened for values that are clearly erroneous (e.g., $0^{\circ}$ or $419^{\circ}$ ); a task that is not trivial.

\subsubsection{Electrogoniometer}

The electrogoniometer system worked reasonably well in the semi-nude condition and for elbow and knee flexions. Even then, sensors must be placed correctly to achieve accurate readings. The low profile of the strain gauges enabled the test garments to slide across the sensors with minimal interference, although the attached cables did require some accommodation. Subjects seldom complained about the sensors and the system was fairly easy to use. 
The electrogoniometer system is relatively expensive and appears to have some technical problems in the application tested in this study. The system is delicate and prone to failure when worn under clothing. As noted above, electrical leads frequently came unplugged from the sensors, and one sensor was even pulled apart during an inadvertent movement by one subject. Numerous readings had to be discarded and the data did not follow the trends clearly evident in the GON and FLEX data as shown in Fig. 6.

\subsection{Absence of a "Gold Standard"}

One of the difficulties encountered in this comparative study was the absence of a "gold standard"; i.e., a well-validated method of ROM measurement that other methods could be compared against. Without a well-validated method for reliably and accurately measuring joint angles, comparisons of ROM test methods must rely on correlation analysis. Positively correlated data sets, such as GON and FLEX, suggest that the techniques operate similarly, but how accurately these methods actually measure ROM remains unanswered.

\section{Summary and recommendation}

The results of this comparative study suggest that the goniometer measurement method be used in future ROM studies involving PPC. Although the accuracy and precision of measurement may be compromised by the inability to visually align the goniometer with joint centers and body segments, we found the goniometer to be as accurate and precise as the Leighton Flexometer and the electrogoniometer when clothing is worn. The goniometer offers the added advantage of being less expensive, easier to use, more sensitive, more durable, and less invasive than the other two measurement techniques evaluated. The electrogoniometer system does not appear to be suited for field or laboratory research involving PPC.

\section{Acknowledgements}

The author would like to thank Lion Apparel for generously providing the garments used in this study, and the University of Michigan Center for Ergonomics for providing the necessary test equipment. This project was partially funded by a training grant provided by the National Institute of Occupational Safety and Health (NIOSH \#OH07207).

\section{Appendix: Criteria for discarding data points}

As mentioned above, numerous electrogoniometer data were discarded. Data points were discarded when:

(1) The angle measurement did not change during movement despite obvious deflection of the sensor blocks (i.e., a measured change of $0^{\circ}$ or $1^{\circ}$ ).

(2) The indicated change in angle during movement suddenly became negative when it clearly should have been positive, or vice versa.

(3) The reading was obviously in error or impossible (e.g., $409^{\circ}$ ).

(4) The sensor was either dislodged from the body surface or open electrical connections were observed. Open connections occasionally occurred when the sensor became disconnected from the wire leading to the amplifier. Sensors are connected to lead wires by small, resistance-fit plugs that could easily be pulled apart during movement.

Discarded data were excluded from all analyses, including standard deviation calculations. Therefore the variance statistics reported for this measurement system do not reflect the true variability of the data. Due to the inconsistency of the data, and the poor correlations with the other measurement methods, detailed analyses among garment treatments were not considered meaningful.

\section{References}

Abraham, S., 1979. Weight and height of adults 18-74 years of age, United States, 1971-1974. Vital and Health Statistics, Series 11, No. 211, Washington D.C.: U.S. Department of Health, Education and Welfare. In: Stephen Pheasant (Ed.), 1986, Bodyspace: Anthropometry, Ergonomics and Design. Taylor and Francis, London. 
Adams, Paul S., 1993. The effects of protective coverall size and fabric weight on wearer range of motion. The Effects of Protective Clothing on Worker Performance: A Study of Size and Fabric Weight Effects on Range-of-Motion, Chapter 4. Unpublished doctoral dissertation, The University of Michigan, Ann Arbor, Michigan.

Alexander, Milton and Laubach, Lloyd, 1973. The effects of personal protective equipment upon the arm-reach capability of USAF pilots. Proceedings Reprint of the Interagency Conference on Management and Technology in the Crew System Process, pp. 225-233.

ASTM Committee F-23 on Protective Clothing, 1988. Standard Practices for Qualitatively Evaluating the Comfort, Fit, Function and Integrity of Chemical Protective Suit Ensembles (F1154-88). American Society for Testing and Materials, Philadelphia, PA.

Bachrach, Arthur J. and Egstrom, Glen H., 1974. Human Engineering Considerations in the Evaluation of Diving Equipment. Naval Medical Research Institute, Bethesda, Maryland (Avail. NTIS: AD-A011 680).

Bensel, Carolyn K., Teixeira, Richard A. and Kaplan, Donna B., 1987. The Effects of U.S. Army Chemical Protective Clothing on Speech Intelligibility, Visual Field, Body Mobility, and Psychomotor Coordination of Men. NATICK/ TR-87/037, U.S. Army Natick Research, Development, and Engineering Center, Natick, Mass. (Avail. NTIS: ADA188 478).

Bishop, Phillip A., Pieroni, Robert E., Smith, Joe F. and Merritt, Thomas W., 1989. Test-retest measurement reliability of human physiological response to heavy work in protective clothing. In: Anil Mital (Ed.), Advances in Industrial Ergonomics and Safety I, pp. 233-237. Taylor and Francis, Philadelphia, PA.

Boone, Donna C., Azen, Stanley P., Lin, Chun-Mei, Spence, Carol, Baron, Carol and Lee, Lynn, 1978. Reliability of goniometric measurements. Physical Therapy, 58(11): $1355-1360$.

Chaffin, Don B. and Andersson, Gurinar B.J., 1991. Occupational Biomechanics, 2nd edition. John Wiley and Sons, New York.

Dunlap and Associates, Inc. and U.S. Army General Equipment Test Activity, 1965. Development of a Methodology for Measuring Infantry Performance in Maneuverability. USATECOM Project No. 8-3-7700-01, U.S. Army General Equipment, Fort Lee, Virginia (Avail. NTIS: AD-467 257).

Faff, Jerzy and Tutak, Tadeusz, 1989. Physiological responses to working with fire fighting equipment in the heat in relation to subjective fatigue. Ergonomics, 32(6): 629-638.

Gamberale, F., 1985. The perception of exertion. Ergonomics, 28(1): 299-308.

Goodwin, J., Clark, C., Deakes, J., Burdon, D. and Lawrence, C., 1992. Clinical methods of goniometry: A comparative study. Disability and Rehabilitation, 14(1): 10-15.

Gregoire, Harv, Call, Douglas W., Coluzzi Omlie, Lisa and Spicuzza, Ronald J., 1985. An automated methodology for conducting human factors evaluations of protective garments. In: Proceedings of the Human Factors Society 29th Annual Meeting, pp. 916-919.

Henry, Maureen S., 1980. Users' Perceptions of Attributes of Functional Apparel. Unpublished master's thesis, Michigan State University, East Lansing, Michigan.
Hollies, Norman R.S., Custer, Anna G., Morin, Catheirne J. and Howard, Marilyn E., 1979. A human perception analysis approach to clothing comfort. Textile Research Journal, 49(10): 557-564.

Huck, Janice, 1988. Protective clothing systems: A technique for evaluating restriction of wearer mobility. Applied Ergonomics, 19(3): 185-190.

Huck, Janice, 1991. Restriction of movement in fire-fighter protective clothing: Evaluation of alternative sleeves and liners. Applied Ergonomics, 22(2): 91-100.

Kirk, William, Jr. and Ibrahim, S.M., 1966. Fundamental relationship of fabric extensibility to anthropometric requirements and garment performance. Textile Research Journal, 36(1): 37-47.

Lotens, W.A., 1986. Loss of Performance Due to Military Clothing and Equipment. IZF-1986-13, Institute for Perception TNO, National Defense, Soesterberg, The Netherlands.

Lotus Development Corporation, 1986. Lotus 1-2-3 ${ }^{\mathrm{TM}}$, Release 2.01. Cambridge, MA: Lotus Development Corporation.

Maxfield, Mary and Smith, Jr., Paul E., 1967. Abbreviated methods for the measurement of oxygen consumption in work physiology. Human Factors, 9(6): 587-594.

Mayerson, N.H. and Milano, R.A., 1984. Goniometric measurement reliability in physical medicine. Archives of Physical Medicine and Rehabilitation, 65: 92-94.

Nicoloff, Christine, 1957. Effects of Clothing on Range of Motion in the Arm and Shoulder Girdle. EP-49, Environmental Protection Research Division, Quartermaster, Natick, Mass. (Avail. NTIS:AD-142 863).

Penny and Giles, Ltd., 1991. Conversation with representative Peter Seddon at the University of Michigan Center for Ergonomics, October.

Petherick, Michele, Rheault, Wendy, Kimble, Sharon, Lechner, Connie and Senear, Virginia, 1988. Concurrent validity and inter-tester reliability of universal and fluid based goniometers for active elbow range of motion. Physical Therapy, 68: 966-969.

Rothstein J.M., Miller, P.J. and Roettger, R.F., 1983. Goniometric reliability in a clinical setting: Elbow and knee measurements. Physical Therapy, 63: 1611-1615.

Saul, Ezra V. and Jaffe, Jack, 1955. The Effects of Clothing on Gross Motor Performance. EP-12, U.S. Army Quartermaster Research and Development Center, Natick, Mass. (Avail. NTIS:AD-066 180).

SAS Institute Inc., 1988. SAS ${ }^{\mathrm{TM}}$ System. Cary, NC: SAS Institute Inc.

Systat, Inc., 1987. SYSTAT ${ }^{\text {TM }}$, Version 3.0. Evanston, IL: Systat, Inc.

Veghte, James H., 1989. The physiologic strain imposed by wearing fully encapsulated chemical protective clothing. In: Jimmy Perkins and Jeffrey Stull (Eds.), Chemical Protective Clothing Performance in Chemical Emergency Response ASTM STP 1037. American Society for Testing and Materials, Philadelphia, PA.

Wick, Charles H., Morrissey, John A. and Klopcic, J. Terrence, 1984. Maintenance Operations in Mission Oriented Protective Posture Level IV (MOPPIV). BRL-MR-3629, U.S. Army Ballistic Research Laboratory, Aberdeen Proving Ground, Maryland (Avail. NTIS: AD-A191 152). 